IJMMS 29:7 (2002) 427-428

PII. S0161171202007962

http://ijmms.hindawi.com

(c) Hindawi Publishing Corp.

\title{
THE CAYLEY TRANSFORM OF BANACH ALGEBRAS
}

\section{ZHIDONG PAN}

\author{
Received 30 July 2001
}

\begin{abstract}
The main result of Haynes (1991) is that a square matrix is convergent $\left(\lim _{n \rightarrow \infty} D^{n}=0\right)$ if and only if it is the Cayley transform $C_{A}=(I-A)^{-1}(I+A)$ of a stable matrix $A$. In this note, we show, with a simple proof, that the above is true in a much more general setting of complex Banach algebras.
\end{abstract}

2000 Mathematics Subject Classification: 15A04, 15A24, 47A60, 47C05.

A square matrix $D$ is called convergent if $\lim _{n \rightarrow \infty} D^{n}=0$, or equivalently, $\lim _{n \rightarrow \infty}\left\|D^{n}\right\|=0$, where $\|\cdot\|$ denotes the matrix norm. A square matrix is called stable if its characteristic values all have negative real parts. The above definitions can be generalized to elements in general complex Banach algebras as follows: let $\mathscr{A}$ be a complex Banach algebra with norm $\|\cdot\|$, and let $\sigma(T)$ be the spectrum of $T$ and $r(T)$ the spectral radius of $T$ for any $T \in \mathscr{A}$. An element $D \in \mathscr{A}$ is called convergent if $\lim _{n \rightarrow \infty}\left\|D^{n}\right\|=0$ and an element $A \in \mathscr{A}$ is called stable if $\sigma(A)$ is contained in the open left half of the complex plane. Inspired by the work of Stein and Taussky [3, 4] and Haynes [2] shows that a square matrix is convergent if and only if it is the Cayley transform of some stable matrix. In this note, we show that this holds for any complex Banach algebra and our proof is much simpler than that of [2].

To this end, we need the following elementary lemma.

LEMMA 1. Let $D \in \mathscr{A}$, then $D$ is convergent if and only if $r(D)<1$.

Proof. $(\Rightarrow)$. First, we show that if $T \in \mathscr{A}$ is convergent then $I-T$ is invertible. Suppose $T$ is convergent, choose a fixed $n$ large enough such that $\left\|T^{n}\right\|<1$. Then $I-T^{n}$ is invertible. Since $I-T^{n}=(I-T) \sum_{k=0}^{n-1} T^{k}$, it follows that $I-T$ is invertible.

Now, note that if $D$ is convergent then so is $(1 / t) D$ for any complex number $t$ such that $|t| \geq 1$. Thus $I-(1 / t) D$ is invertible. Therefore, $t I-D$ is invertible. This implies that $r(D)<1$.

$(\Leftarrow)$. Suppose $r(D)<1$. Then choose a fixed $\epsilon>0$ such that $r(D)+\epsilon<1$. For $n$ large enough, we have $\left\|D^{n}\right\|^{1 / n}<r(D)+\epsilon$. Thus, $\left\|D^{n}\right\|<(r(D)+\epsilon)^{n}$. Therefore, $D$ is convergent.

For any stable element $A \in A, I-A$ is invertible. In this case, we define the Cayley transform of $A$ as $C_{A}=(I-A)^{-1}(I+A)$.

THEOREM 2. For any complex Banach algebra $\mathscr{A}$, an element $D \in \mathscr{A}$ is convergent if and only if $D=C_{A}$ for some stable $A \in A$.

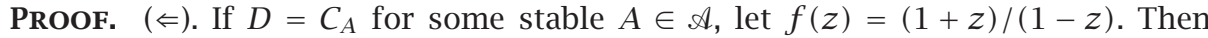
$f(z)$ is analytic in the open left half of the complex plane. In fact, $f(z)$ is a Möbius 
transform that maps the open left half of the complex plane onto the open unit disk and $f(A)=C_{A}$. Since $f(z)$ is analytic in a neighborhood of $\sigma(A)$. By the Spectral Mapping theorem (see [1, page 208]), $\sigma(f(A))=f(\sigma(A))$. Since $f(\sigma(A))$ is contained in the open unit disk, $\sigma(D)=\sigma(f(A))$ is contained in the open unit disk. By Lemma 1 , $D$ is convergent.

$(\Rightarrow)$. Suppose $D$ is convergent. By Lemma $1, \sigma(D)$ is contained in the open unit disk. Thus $D+I$ is invertible. Let $A=(D+I)^{-1}(D-I)$. Define $g(z)=(z-1) /(z+1)$. The map $g(z)$ is analytic in the open unit disk. In fact, $g(z)$ is a Möbius transform that maps the open unit disk onto the open left half of the complex plane and $g(D)=A$. Similarly, by the Spectral Mapping theorem, we can obtain $\sigma(A)=\sigma(g(D))=g(\sigma(D))$. This implies that $\sigma(A)$ is contained in the open left half of the complex plane, that is, $A$ is stable. Solving for $D$ from $A=(D+I)^{-1}(D-I)$, we have $D=(I-A)^{-1}(I+A)$, that is, $D=C_{A}$.

\section{REFERENCES}

[1] J. B. Conway, A Course in Functional Analysis, Graduate Texts in Mathematics, vol. 96, Springer-Verlag, New York, 1985.

[2] T. Haynes, Stable matrices, the Cayley transform, and convergent matrices, Int. J. Math. Math. Sci. 14 (1991), no. 1, 77-81.

[3] P. Stein, On the ranges of two functions of positive definite matrices, J. Algebra 2 (1965), 350-353.

[4] O. Taussky, Matrices $C$ with $C^{n} \rightarrow 0$, J. Algebra 1 (1964), 5-10.

Zhidong Pan: Department of Mathematics, Saginaw Valley STAte University, UNIVERSITY CENTER, MI 48710, USA

E-mail address: pan@svsu.edu 


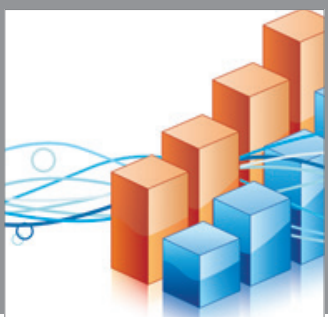

Advances in

Operations Research

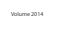

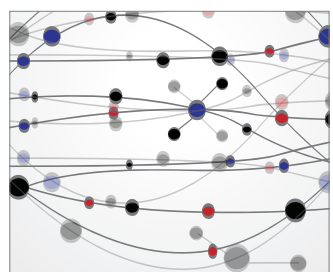

\section{The Scientific} World Journal
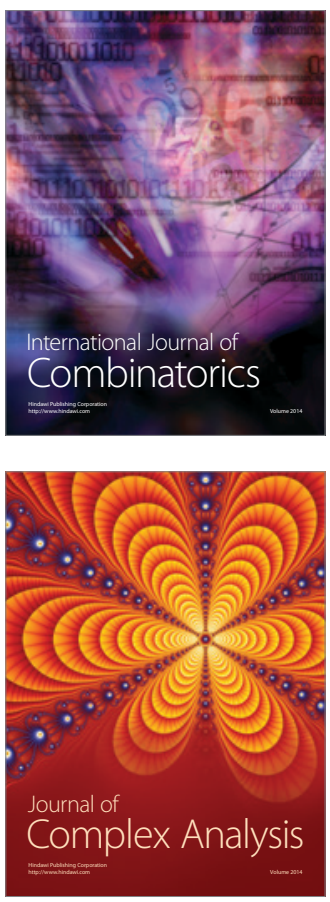

International Journal of

Mathematics and

Mathematical

Sciences
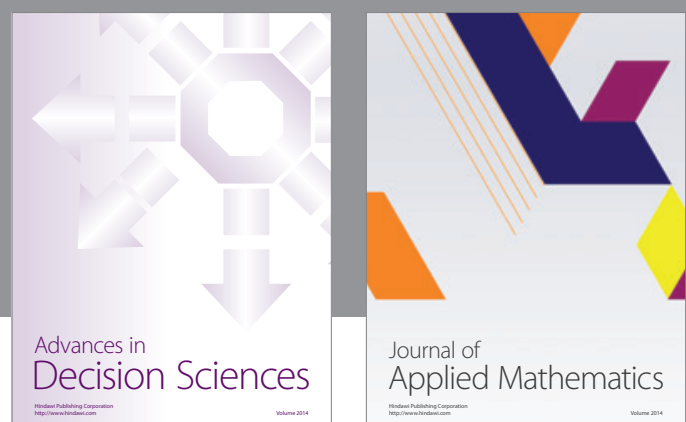

Journal of

Applied Mathematics
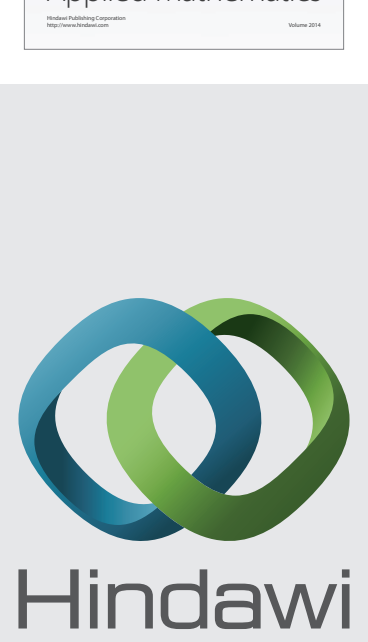

Submit your manuscripts at http://www.hindawi.com
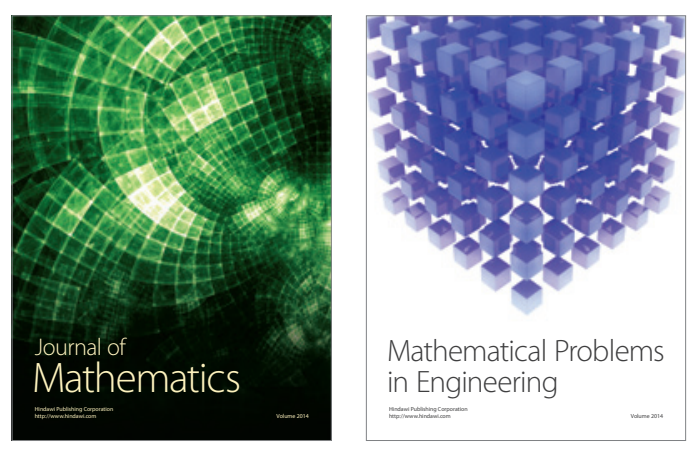

Mathematical Problems in Engineering
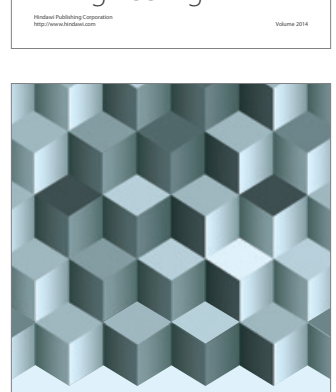

Journal of

Function Spaces
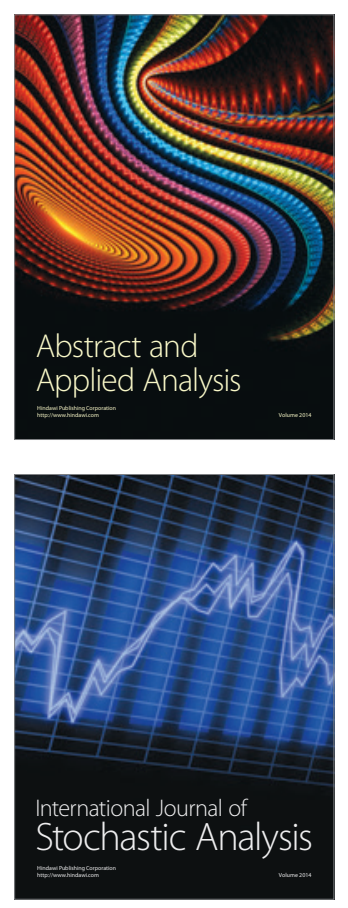

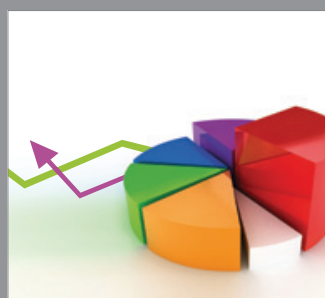

ournal of

Probability and Statistics

Promensencen
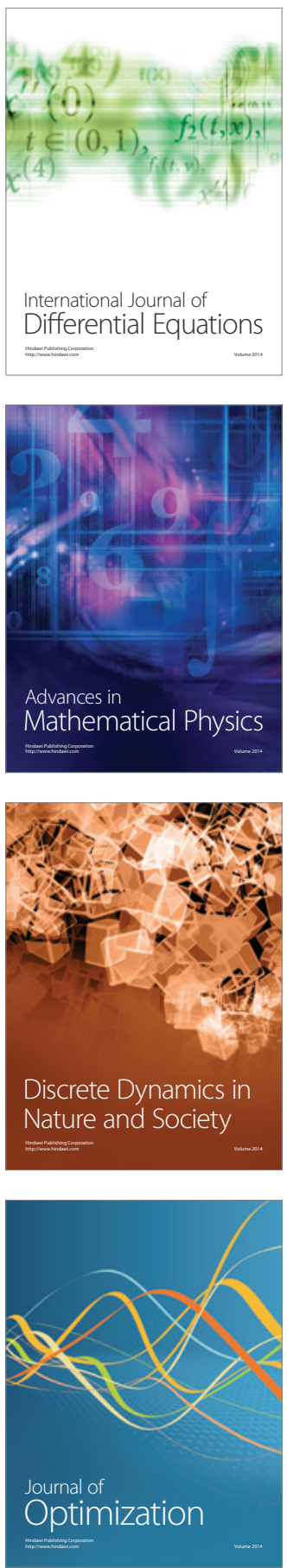